\title{
Position Detection Using One Photodiode and Multiple LED Makers
}

\author{
Kosuke Onishi, Nobuhiro Hirano, Shiyuan Yang \\ Kyushu Institute of Technology, Japan \\ *Corresponding Author: p349407k@mail.kyutech.jp
}

\begin{abstract}
This paper describes a new approach for controlling an autonomous mobile robot indoors. In recent years, many studies are being done in order to solve the problem of an autonomous mobile robot indoors. The position detection of a mobile robot requires many artificial landmarks. Thus, the conventional method have to create landmark database which is stored large amount of landmark data. As a result, the conventional method takes much time to detect the position of a mobile robot. Moreover, preparation of many artificial markers is a cumbersome task for the user. Therefore, we propose the new landmark system which is able to detect position simply, quickly and accurately.

In recent years, Light Emitting Diode (LED) lighting is spreading rapidly as an indoor lighting. So, the proposal method uses frequency modulated LED lightings as landmarks indoors. It can distinguish between LED lightings quickly and accurately by difference of the frequency. The measurement system consists of a light projecting unit and a light receiving unit. The light projecting unit consists of a frequency modulation circuit and LEDs. The light receiving unit can detect the position of the mobile robot quickly from the receiving amount of a photodiode mounted on the mobile robot without the image processing.

We verified the accuracy of the position detection. The proposal method could distinguish between multiple LED lightings accurately. In addition, the maximum error of the position detection was $2.70 \mathrm{~cm}$. The maximum error rate was $1.45 \%$. The accuracy was considerably high. Measurement time was only $55 \mathrm{msec}$. The efficacy of the proposal method was confirmed by the experiment.
\end{abstract}

Keywords: position detection, photodiode, LED.

\section{Introduction}

In recent years, many studies are being done in order to solve the problem of autonomous mobile robot indoors. The position detection of a mobile robot requires many environment information.

There are methods using a Global Positioning System (GPS) or a camera as the position detection. Various techniques that employ GPS signals such as A-GPS and GPS transmitters, have been introduced with the hope to provide a solution for indoor positioning detection ${ }^{(1)}$. The GPS system can detect the position everywhere if the wireless communication is possible. However, GPS system error gets dozens meters worse at indoor or urban canyon where radio wave is hard to reach $^{(2)}$. Therefore, the indoor position detection often uses a camera.

The camera system requires the landmark data and the map data of building previously. The camera system detects position by collating the filmed image and the landmark data in the map data. Large amount of landmark data is stored in the landmark database, so that the camera system takes much time to collate the filmed image and the landmark data. Moreover, the camera system needs to allocate many artificial markers otherwise an estimation error will accumulate. Thus, it is difficult to use such methods in large and natural environments ${ }^{(3)}$. Preparation of many artificial markers is a cumbersome task for the user. Furthermore, a camera is much more expensive than a photodiode.

In this paper, we propose the position detection

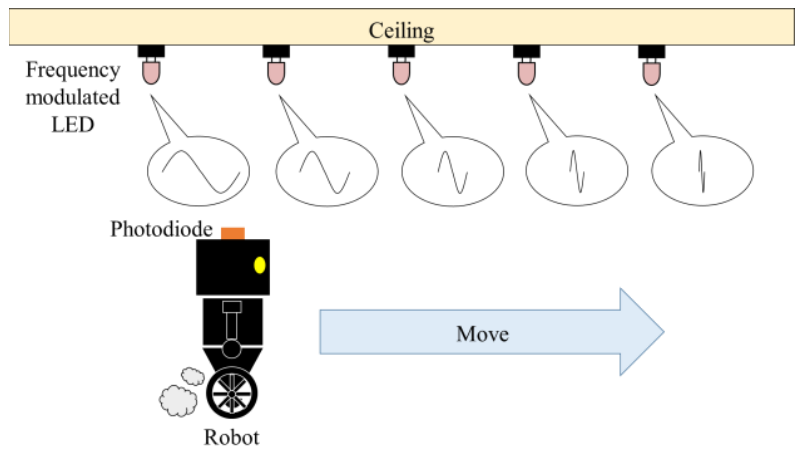

Fig. 1. System schematic diagram. 
method using one photodiode and multiple LED markers. Fig. 1 shows system schematic diagram. The characteristics of the proposal method are as follows:

- Amount of available landmark is many.

- Landmark recognizing is quick and accurate.

- Position detection speed is quick and simple.

- Measurement system is low prices.

The proposal method uses frequency modulated LED markers. The frequency of a fluorescent lamp is $60 \mathrm{~Hz}$. However, a fluorescent lamp does not affect the human body. In recent years, various studies on the visible light communication are conducted. The visible light communication is wireless technology using frequency modulated LEDs. It has the following characteristics. First, the peripheral electronics are not adversely because electromagnetic noises are not generated affected. Second, the modulated light does not affect the human body. Therefore, the proposal method also does not affect the human body.

\section{Measurement system}

Fig.2 shows the setup of the measurement system we made actually. The system consist of a light projecting unit and a light receiving unit. Fig. 3 shows the flow of the measurement system.

\subsection{Light projecting unit}

The light projecting unit projects light which the

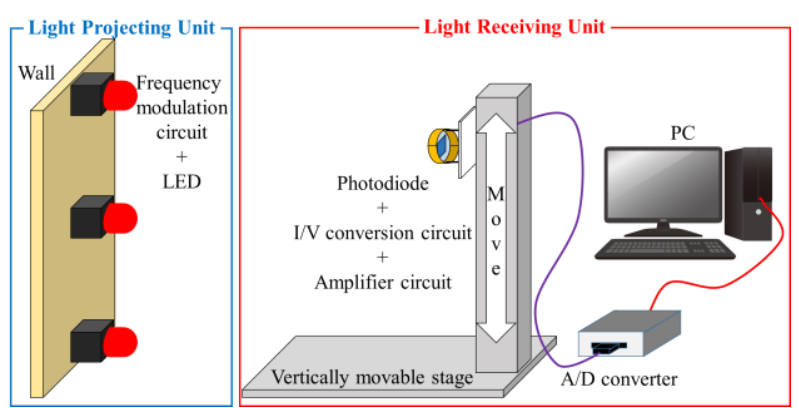

Fig. 2. Setup of the measurement system.

frequency is modulated. The unit consist of a frequency modulation circuit and LEDs. The frequency modulation circuit modulates frequency of LEDs so that the light receiving unit can distinguish light signal of LEDs or reduce the influence of the disturbance light.

\subsection{Light receiving unit}

The light receiving unit consist of a photodiode, an I/V conversion circuit, an amplifier circuit and PC. A photodiode of the light receiving unit receives light of the light projecting unit. The photodiode outputs the current in response to the amount of light received.

The I/V conversion circuit converts the current to the voltage. The amplifier circuit amplifies the voltage because the voltage is very small. Moreover, the amplifier circuit can reduce the influence of disturbance light by eliminating the voltage of unnecessary frequency band.

The amplified voltage is inputted to the PC after $\mathrm{A} / \mathrm{D}$ conversion. The inputted data is the synthesized wave

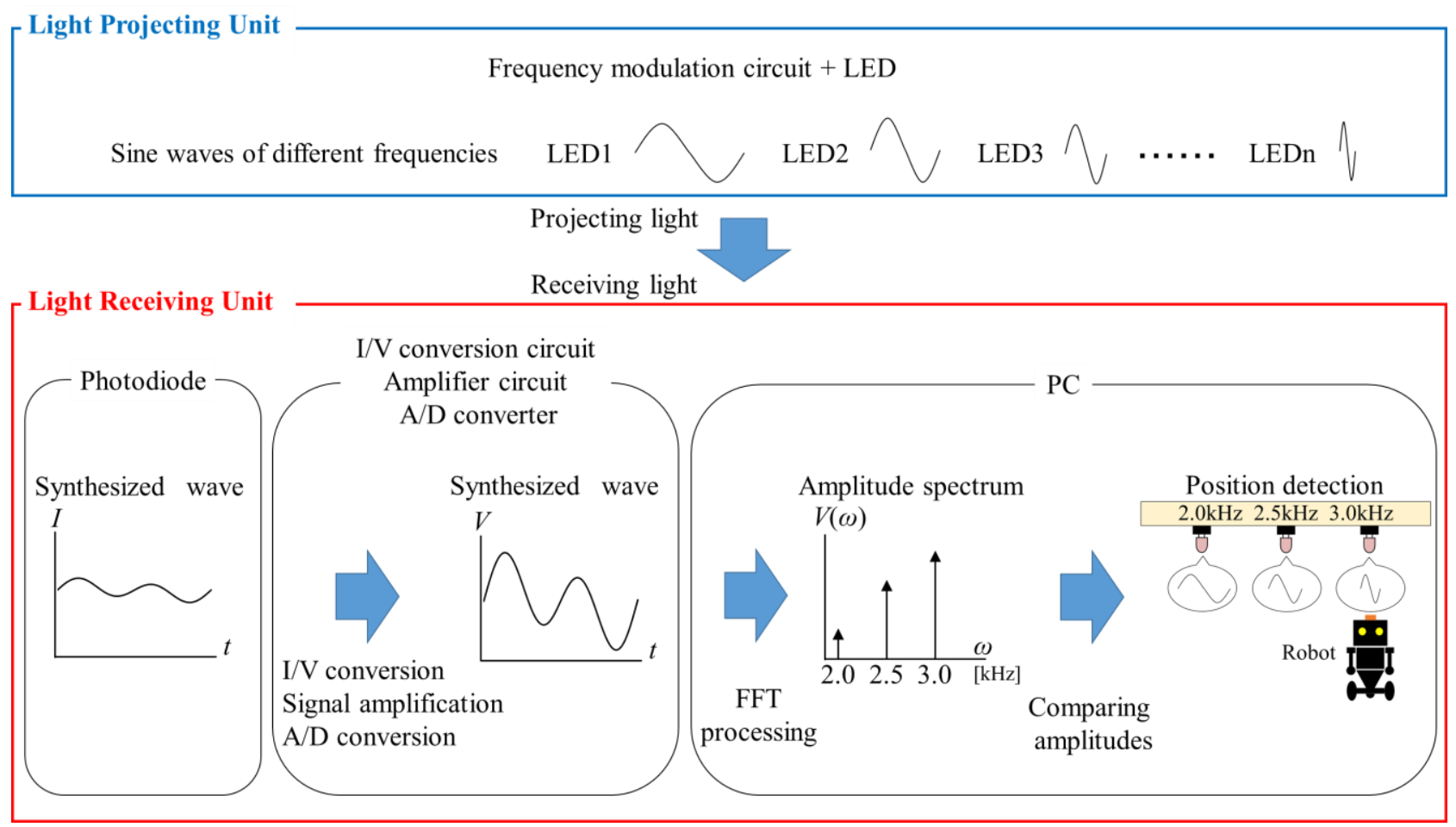

Fig. 3. Flow of measurement system. 
including the light signal of multiple LEDs. Therefore, the inputted data must be converted to the amplitude spectrum data representing an amplitude spectrum distribution about a frequency axis by the FFT processing. The amplitude of the amplitude spectrum data is fluctuated in response to the amount of light received by a photodiode. As a result, the position of the light receiving unit is detected by using the frequency and the amplitude of the amplitude spectrum data

\section{Experiment}

\subsection{Experimental device}

(a) Light projecting unit

In this experiment, OST10WG01GD-4WM387E1U was used as LED lighting. Table.1 shows the size and the electrical-optical characteristics of the LED.

Table. 1 Specifications of LED OST10WG01GD.

\begin{tabular}{ll}
\hline Spec & Value \\
\hline Diameter & $8 \mathrm{~mm}$ \\
Wavelength & $5500-8000 \mathrm{~K}$ \\
Overall Luminous Flux & $551 \mathrm{~m}$ \\
Directivity & $140^{\circ}$ \\
LED Module Power & $0.48 \mathrm{~W}$ \\
Offset Voltage & $11 \mathrm{~V}$ \\
Amplitude & $1.3 \mathrm{~V}$ \\
\hline
\end{tabular}

Fig.4 shows the circuit diagram of the frequency modulation we made. From Fig.4, XR-2206 was used as the monolithic function generator. The frequency modulation circuit could generate various signal by changing the resistance value.

(b) Light receiving unit

In this experiment, S12698-02 was used as a photodiode. Table. 2 shows the size and the electrical-optical

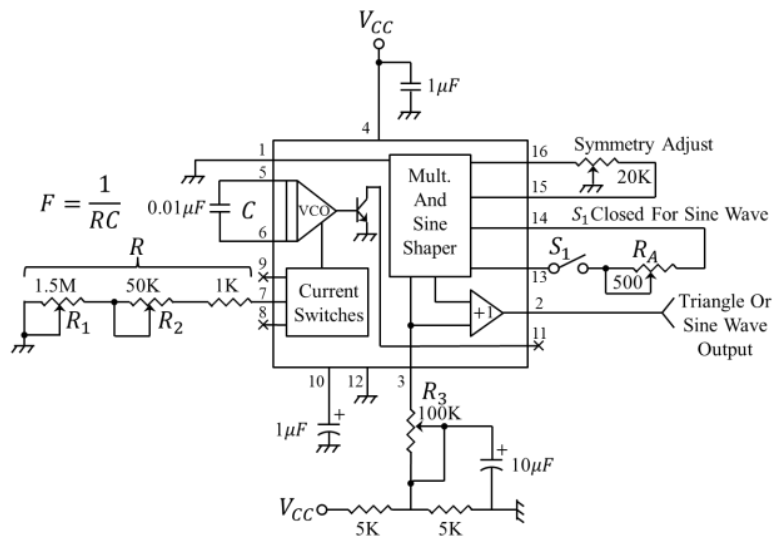

Fig. 4. Circuit diagram of the frequency modulation. characteristics of the photodiode. Operational amplifier NJM4580DD was used as the I/V conversion and amplifier circuit. TUSB-1612ADSM was used as the A/D convertor. Table. 3 shows Specifications of the PC.

Table. 2 Specifications of photodiode S12698-02.

\begin{tabular}{ll}
\hline Spec & Value \\
\hline Resolution & $1 / 4096(12 \mathrm{bit})$ \\
Conversion Error & $\pm 4 \mathrm{LSB}$ \\
Conversion Rate & $100 \mathrm{kHz}$ \\
Input Voltage & $-12 \mathrm{~V} \sim+12 \mathrm{~V}$ \\
\hline
\end{tabular}

Table. 3 Specifications of A/D convertor TUSB-1612ADSM.

\begin{tabular}{ll}
\hline Spec & Value \\
\hline Photosensitive Area Size & $5.8 \times 5.8 \mathrm{~mm}$ \\
Effective Photosensitive Area & $33.6 \mathrm{~mm}^{2}$ \\
Spectral Response Range & 190 to 1000 \\
Directivity & $140^{\circ}$ \\
\hline
\end{tabular}

\subsection{Experimental method}

We verified the accuracy of the position detection. Experimental location was under fluorescent lamps. The frequency of LED1, LED2, LED3 was $2.0 \mathrm{kHz}, 2.5 \mathrm{kHz}, 3.0$ $\mathrm{kHz}$ to prevent the influence of the fluorescent lamps.

Fig. 5 shows the positional relationship of LEDs and the photodiode. In this experiment, LEDs were installed in $50 \mathrm{~cm}$ intervals on the wall. The position of LED1, LED2, LED3 was $-50 \mathrm{~cm}, 0 \mathrm{~cm},+50 \mathrm{~cm}$. A photodiode of the light receiving unit was installed a vertically movable stage. An interval between the wall and the photodiode was $2.0 \mathrm{~m}$. The photodiode was moved from $+25 \mathrm{~cm}$ to $-25 \mathrm{~cm}$. The position was detected in $5 \mathrm{~cm}$ intervals. This detection was repeated 3 times to verify the variation of the detected positions.

\subsection{Experimental result}

Fig. 6 shows the amplitude of the amplitude spectrum

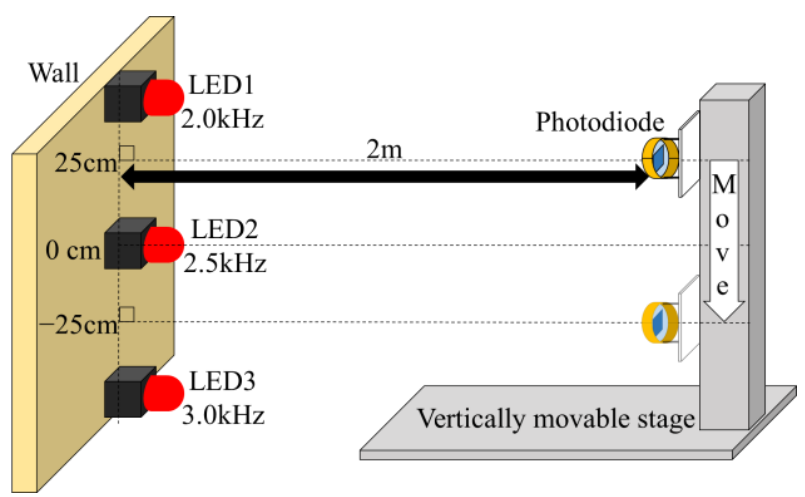

Fig. 5. Position of LEDs and a photodiode. 


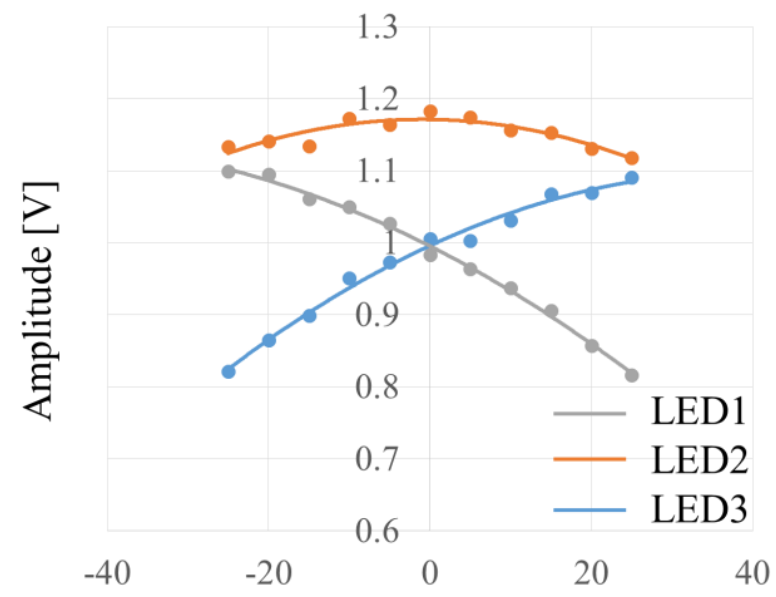

Photodiode position[cm]

(a) First detection.

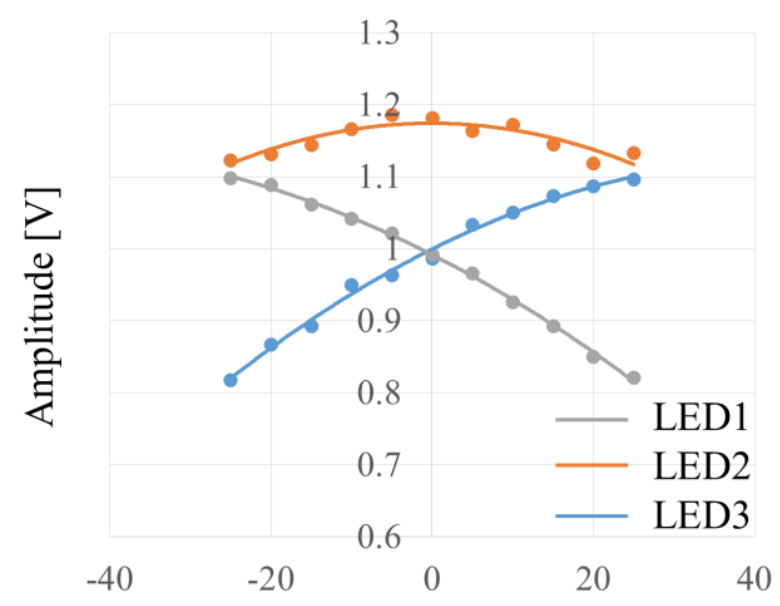

Photodiode position[ $\mathrm{cm}$ ]

(b) Second detection.

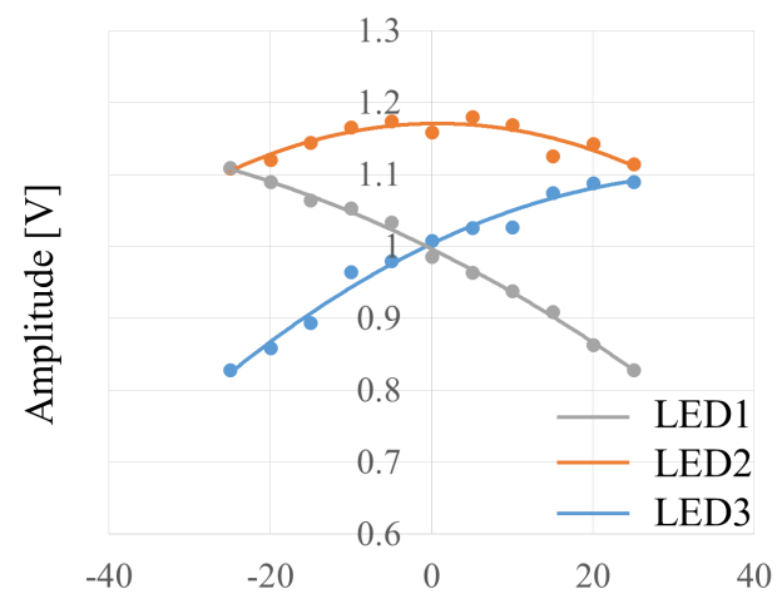

Photodiode position[ $\mathrm{cm}]$

(c) Third detection.

Fig. 6. Amplitude of the amplitude spectrum data in 3 times detection. data in $2.0 \mathrm{kHz}, 2.5 \mathrm{kHz}$, and $3.0 \mathrm{kHz}$. The change of the amplitude was a curved line. The amplitude was large when the distance between the photodiode and the LED was near. On the other hand, the amplitude was small when the distance between the photodiode and the LED was far. As a result, the measurement system could detect the position by detecting the maximum amplitude. The interval of LEDs determined the range of error. In this experiment, The accuracy of the position detection was $\pm 25 \mathrm{~cm}$ because the interval of LEDs was $50 \mathrm{~cm}$. In addition, Fig. 7 shows the variation of the amplitude as the standard deviation in 3 times detection. The maximum standard deviation was $0.013 \mathrm{~V}$. These values were very small.

The amplitudes of multiple LEDs were compared to improve the accuracy of the position detection. The change of the amplitude was large when the distance between the photodiode and the LED was far. Therefore, the amplitudes of LED1 and LED3 were available for the position detection because those had linearity. Fig. 8 shows the principle of the position detection. Equation 1 was used to detect the position.

$$
y=\frac{V 1-V 2}{V 1+V 2-2 V \min } \times \frac{c}{2}
$$

The $y$ was the photodiode position. The $V I$ was the amplitude of the LED1. The $V 2$ was the amplitude of the LED3. The $c$ was the distance between the LED1 and LED3. Fig. 9 shows the result of the position detection based on the amplitudes of LED1 and LED3. The measurement value almost coincided with the theoretical value. The maximum error was $2.70 \mathrm{~cm}$. The maximum error rate was $1.45 \%$. As a result, the accuracy of the position detection was improved

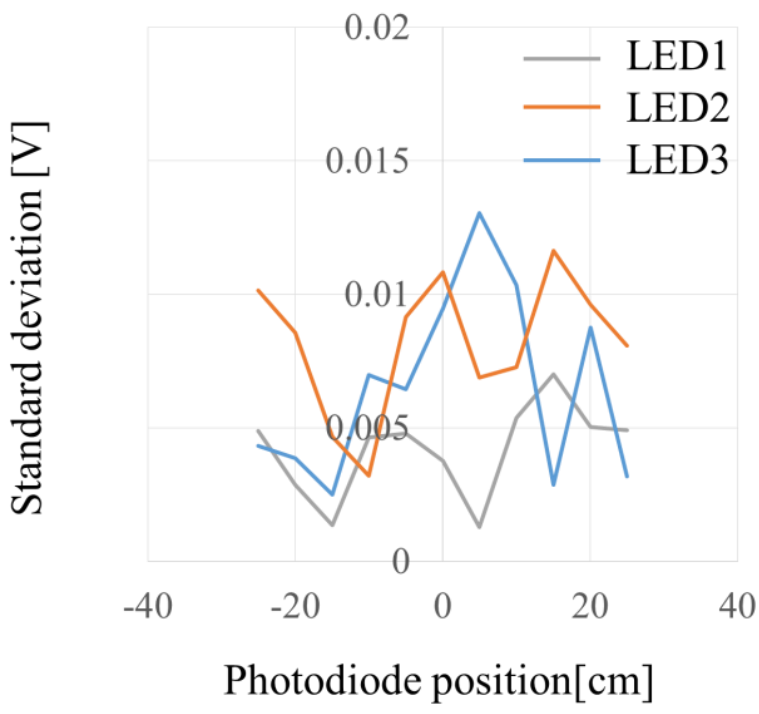

Fig. 7. Standard deviation in 3 times detection. 


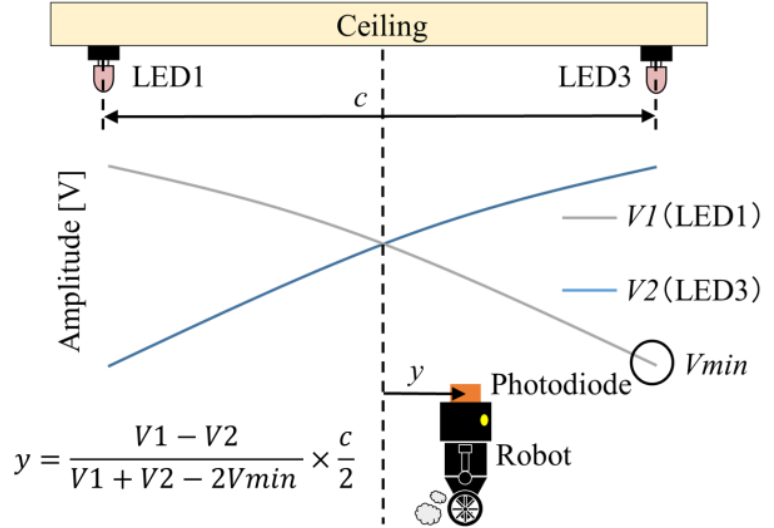

Fig. 8. Principle of the position detection.

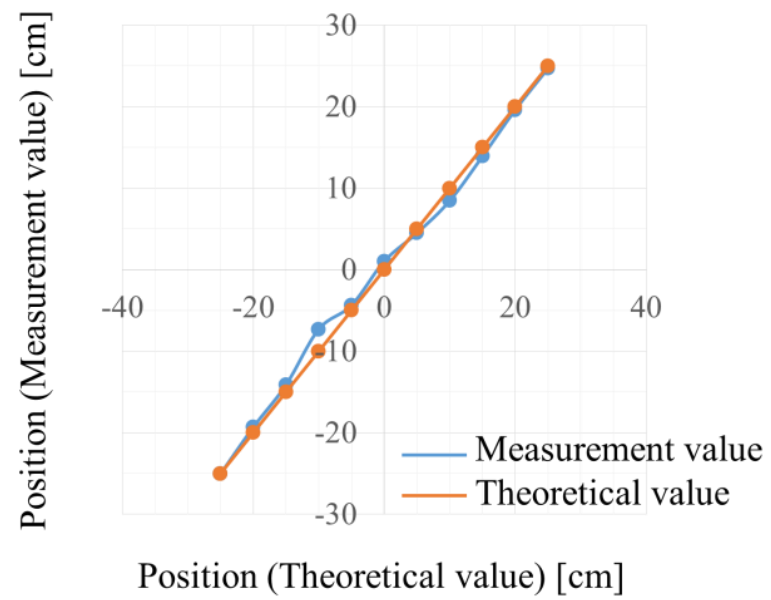

Fig. 9. Result of the position detection.

by comparing the amplitudes of multiple LEDs. Measurement time was $55 \mathrm{msec}$. From the above, the proposal method was an effective the method of position detection.

\section{Conclusions}

The proposal method used frequency modulated LED lightings indoors as landmarks, so that it could treat more landmarks simply than before. Moreover, it could recognize the LEDs as the landmark quickly and accurately. Furthermore, it could detect position of a photodiode mounted on the mobile robot. In addition, the proposal method is low prices because it is not used costly devices such as a camera and a PSD.

Our future work is improvement of position detection and proposal of 3 dimensional position detection method using multiple photodiode and multiple ceiling LED lighting. Moreover, the proposal method has a condition that the photosensitive area of a photodiode must be arranged in parallel to ceiling. Therefore, our future work is resolution of the problem.

\section{References}

(1) Nor Aida Mahiddin, Elissa Nadia Madi, Siti Dhalila, Engku Fadzli Hasan, Suhailan Safie and Noaizan Safie : "User Position Detection In An Indoor Environment.", International Journal of Multimedia and Ubiquitous Engineering, Vol.8, No.5, pp.303-312, 2013

(2) Yoshino Masaki, Shinichiro Haruyama, Masao Nakagawa : "High-accuracy positioning system using visible LED lights and image sensor.” 2008 IEEE Radio and Wireless Symposium. IEEE, pp.439-442, 2008.

(3) Oe, Motoko, Tomokazu Sato, and Naokazu Yokoya : "Estimating camera position and posture by using feature landmark database." Scandinavian Conference on Image Analysis. Springer Berlin Heidelberg, pp.171$181,2005$.

(4) Z. Barbaric : "Sensitivity Analysis of a Position Device with Quadrant Photodiode.", Scientific Publications of the State University of Novi Pazar, Vol. 5, No. 2, pp.8592, 2013

(5) Kosuke Onishi, Daisuke Tanaka, Shiyuan Yang : “3D Position Detection Using a 2D PSD and a Marker Composed of Light Sources.", Journal of the Institute of Industrial Applications Engineers, Vol.4, No2, pp.86-93, 2016 